\title{
Proteomics-based analysis indicating $\alpha$-enolase as a potential biomarker in primary Sjögren's syndrome
}

\author{
Pan Wei ${ }^{1}$, Yixiao Xing ${ }^{1}$, Boya $\mathrm{Li}^{1}$, Feng Chen ${ }^{2}$, Hong Hua ${ }^{1}$ \\ ${ }^{1}$ Department of Oral Medicine, Peking University School and Hospital of Stomatology, Beijing, China; ${ }^{2}$ Central Laboratory, Peking University \\ School and Hospital of Stomatology, Beijing, China \\ Contributions: (I) Conception and design: P Wei, F Chen, H Hua; (II) Administrative support: H Hua; (III) Provision of study materials or patients: \\ P Wei, H Hua; (IV) Collection and assembly of data: All authors; (V) Data analysis and interpretation: All authors; (VI) Manuscript writing: All \\ authors; (VII) Final approval of manuscript: All authors. \\ Correspondence to: Feng Chen. Central Laboratory, School and Hospital of Stomatology, Peking University, No. 22 Zhongguancun South Avenue, \\ Beijing 100081, China. Email: molecularstone@gmail.com; Hong Hua. Department of Oral Medicine, School and Hospital of Stomatology, Peking \\ University, No. 22 Zhongguancun South Avenue, Beijing 100081, China. Email: Honghua1986@aliyun.com.
}

Background: Primary Sjögren's syndrome (pSS) is a chronic autoimmune disease. Its etiology is not well understood. Salivary glands are the main target organ in pSS, investigating the changes of salivary protein in pSS patients may not only be a valuable way of identifying new biomarkers/antigens for pSS, but also of revealing the pathogenesis underlying this autoimmune disease. In the present study, we aimed to investigate new biomarkers and explore their potential role in pSS.

Methods: In this study, $\alpha$-enolase (ENO1) was found to be overexpressed in pSS by $1 \mathrm{D}$ gel electrophoresis/ mass spectrometry. The finding was verified by Western blots, immunohistochemistry (IHC), and polymerase chain reaction (PCR) results in both saliva and labial salivary glands. The expression level of immunoglobulin $\mathrm{G}(\mathrm{IgG})$ antibody to ENO1 was then tested by enzyme-linked immunosorbent assay (ELISA).

Results: ENO1 autoantibody was found to be overexpressed in pSS compared with healthy controls. The effects of ENO1 overexpression on rat submandibular gland cell line SMG-C6 was investigated in vitro. The expressions of proteins related to saliva secretion and immunomodulatory were upregulated in ENO1 overexpressed SMG-C6 cells.

Conclusions: Both ENO1 and anti-ENO1 autoantibody are overexpressed in pSS patients. Nevertheless, their potential role in the pathogenesis of pSS warrants further study.

Keywords: Primary Sjögren's syndrome (pSS); $\alpha$-enolase (ENO1); proteomics-based analysis; mass spectrometry

Submitted Sep 23, 2020. Accepted for publication Dec 13, 2020.

doi: 10.21037 /gs-20-814

View this article at: http://dx.doi.org/10.21037/gs-20-814

\section{Introduction}

Primary Sjögren's syndrome (pSS) is a chronic autoimmune disease characterized by dry mouth/eye as a result of immune-mediated injury of exocrine glands. The onset of pSS is insidious, with a variable course and a diversity of clinical manifestations, making the diagnosis difficult or delayed. Research has shown that the diagnosis of pSS is usually delayed by nearly 6 years from onset $(1,2)$. The etiology of pSS is far from clear. Several factors, such as injury, viruses, and/or self-antigen might initiate the progression of this autoimmune disease $(3,4)$.

Several studies have demonstrated that antigenic changes are observed in the immune system of patients, including the appearance of circulating autoantibodies (5). To date, various serum antibodies, such as anti-Ro/SSA, anti-La/ $\mathrm{SSB}$, anti-muscarinic receptor antibodies, anti-nuclear antibodies, and rheumatoid factor, have been reported as characteristics of pSS (6), with some having a pathogenic 
role (e.g., anti-Ro/SSA and anti-muscarinic receptor antibodies) (7). Therefore, investigations of new pSS biomarkers/antigens could contribute to both the diagnosis and understanding of pathogenesis of this autoimmune disorder.

Saliva is a mixed fluid secreted by 3 pairs of major salivary glands. Previous research has demonstrated that saliva contains many informative molecules that can reflect the entire spectrum of health and disease states of an individual (8). As salivary glands are the main target organ in pSS, investigating the changes of salivary protein in pSS patients may not only be a valuable way of identifying new biomarkers/antigens for pSS, but also of revealing the pathogenesis underlying this autoimmune disease.

In the present study, we screened a series of overexpressed proteins, including $\alpha$-enolase (ENO1) in the saliva of pSS patients, using 1D gel electrophoresis/ mass spectrometry. The expression of ENO1 was verified in saliva and labial glands, and the levels of serum antibody to ENO1 were measured. To investigate the potential pathogenetic role of ENO1, ENO1 plasmid was transfected into rat submandibular gland cell line SMG-C6. Protein expression was detected by mass spectrometry in the ENO1 overexpressed SMG-C6 cell. Our results indicated that ENO1 and its antibody may serve as biomarkers for pSS, and the overexpression may be related to salivary gland hypofunction and immune inflammatory response.

We present the following article in accordance with the MDAR checklist (available at http://dx.doi.org/10.21037/ gs-20-814).

\section{Methods}

\section{Patients and specimen collection}

The present study was approved by the Biomedical Ethics Committee of Peking University School of Stomatology (Beijing, China). All procedures performed in this study involving human participants were in accordance with the Declaration of Helsinki (as revised in 2013). All participants provided signed informed consent. Patients with a diagnosis of pSS, according to the 2002 American-European Consensus Group (AECG) criteria (6), were enrolled from the outpatient clinics of the Department of Oral Medicine, Peking University Hospital of Stomatology (Beijing, China). All patients were newly diagnosed and had not undergone treatment. Saliva samples were collected and disposed of, as previously described (9), from 10 pSS patients and 10 healthy controls for mass spectrometry analysis and Western blot validation of ENO1 expression. Serum samples were collected from 30 pSS patients and 30 healthy controls, and separated by centrifugation at $1,006 \times \mathrm{g}$ for $10 \mathrm{~min}$ and stored at $-80{ }^{\circ} \mathrm{C}$ for anti-ENO1 antibody detection. Labial glands were obtained by labial biopsy from $5 \mathrm{pSS}$ patients and 4 healthy controls (otherwise healthy patients with patients with mucous cysts). Control labial glands were histologically normal, with no lymphocyte infiltration foci. Labial glands were stored at $-80{ }^{\circ} \mathrm{C}$ for polymerase chain reaction (PCR) sequencing, and fixed using $10 \%$ formaldehyde solution, and embedded in paraffin for immunohistochemistry (IHC).

\section{Protein separation by $1 D$ sodium dodecylsulfate- polyacrylamide gel electrophoresis (SDS-PAGE) and proteomics analysis}

Saliva samples from $10 \mathrm{pSS}$ patients and 10 controls were separately pooled into 2 groups. Approximately $30 \mu \mathrm{g}$ proteins from each pooled samples were separated by $1 \mathrm{D}$ SDS-PAGE. The gel bands were excised from the gel and reduced with $25 \mathrm{mM}$ of dithiothreitol and alkylated with $55 \mathrm{mM}$ iodoacetamide. Sequencing grade modified trypsin in $50 \mathrm{mM}$ ammonium bicarbonate was used for ingel digestion at $37{ }^{\circ} \mathrm{C}$ overnight. The peptides were then extracted twice with $0.1 \%$ trifluoroacetic acid in $50 \%$ acetonitrile aqueous solution for $30 \mathrm{~min}$. Extracts were then centrifuged in a SpeedVac (Thermo Fisher Scientific, Waltham, MA, USA) to reduce the volume.

The digestion products were separated by a $65-\mathrm{min}$ gradient elution with a flow rate of $0.250 \mathrm{~mL} / \mathrm{min}$ for mass spectrometry analysis. The liquid chromatography with tandem mass spectrometry (LC-MS/MS) analysis was then carried out with an EASY-nLCIITM integrated nanoHPLC system and a directly interfaced Thermo Scientific LTQ Orbitrap mass spectrometer (Waltham, MA, USA). The analytical column was a fused silica capillary column (75 mm ID, 150 mm length; Upchurch, Oak Harbor, WA, USA) filled with $\mathrm{C}-18$ resin $\left(300 \mathrm{~A}^{\circ}, 5 \mathrm{~mm}\right.$; Varian, Lexington, MA, USA). Mobile phase A consisted of $0.1 \%$ formic acid, and mobile phase B consisted of $100 \%$ acetonitrile and $0.1 \%$ formic acid. The mass spectrometry analysis and database search were performed as previously described (10).

\section{Western blot}

Saliva samples from 10 pSS patients and 10 healthy 
controls were centrifuged at $14,000 \times \mathrm{g}$ for $10 \mathrm{~min}$ at $4{ }^{\circ} \mathrm{C}$ for supernatant collection. Protein concentrations were determined using the bicinchoninic acid (BCA) protein assay kit. Protein samples were separated with a $12 \%$ SDS-PAGE gel and transferred onto a polyvinylidene difluoride (PVDF) transfer membrane by electroblotting. After blocking with $5 \%$ non-fat milk in Tris-buffered saline Tween 20, the membrane was incubated with primary antibody at $4{ }^{\circ} \mathrm{C}$ overnight and horseradish peroxidase-labeled secondary antibody at room temperature for $2 \mathrm{~h}$. The membrane was further washed with PBST buffer 3 times and developed using the Enlight Kit (Engreen, Beijing, China). Glyceraldehyde 3-phosphate dehydrogenase (GAPDH) was detected as an internal control.

\section{Quantitative real-time PCR}

Labial glands were collected from pSS patients and otherwise healthy patients with mucous cysts as healthy controls. Total RNA was extracted using TRIzol reagent (Life Technologies, Carlsbad, CA, USA). cDNA was synthesized from $0.8 \mu \mathrm{g}$ RNA using the GoScript Reverse Transcription System (Promega, Beijing, China). Quantitative PCR was performed using the ABI7500 (Applied Biosystems, Foster City, CA, USA) PCR system with UltraSYBR Mixture (category no. CW0956; CoWin BioSciences, Cambridge, MA, USA). The primer was designed and synthesized by CoWin BioSciences. The forward and reverse primers for ENO1 were 5'-CAGAGTTTCGCCCGCACCAC-3' and 5'-GGGAGAGCCGTCACTCATTC-3', respectively. The length of the PCR products was $159 \mathrm{bp}$, with GAPDH used as an internal control. Amplification was performed at $95{ }^{\circ} \mathrm{C}$ for $10 \mathrm{~min}\left(95^{\circ} \mathrm{C}\right.$ for $15 \mathrm{~s}+60{ }^{\circ} \mathrm{C}$ for $\left.60 \mathrm{~s}\right)$ for 40 cycles. The Comparative CT Method $\left(2^{-\triangle \Delta C T}\right)$ method was used for relative quantitative data analysis.

\section{IHC}

Labial gland specimens from pSS patients and healthy controls were embedded in paraffin and sectioned at $4 \mu \mathrm{m}$. After deparaffinization and rehydration using descending ethanol dilutions, antigen retrieval was performed with boiling citrate buffer $(\mathrm{pH}$ 6.0) in a microwave. Endogenous peroxidases were then quenched with 3\% $\mathrm{H}_{2} \mathrm{O}_{2}$ for $15 \mathrm{~min}$. The sections were then incubated with anti-ENO1 Ab (1:100; ABclonal, Wuhan, China) at $4{ }^{\circ} \mathrm{C}$ overnight and stained using a tissue staining kit
(Zhongshan Biotechnology, Beijing, China) according to the manufacturer's protocol. The stained sections were mounted and examined by microscopy. Representative sections were photographed.

\section{Enzyme-linked immunosorbent assay (ELISA)}

Serum samples were collected from 26 pSS patients and 25 healthy controls. The level of serum immunoglobulin G (IgG) anti-ENO1 autoantibody was detected by an ELISA kit (Jianglai Biology, Shanghai, China) according to the manufacturer's protocol.

\section{Cell culture and ENO1 overexpression}

The rat submandibular gland cell line SMG-C6 (kindly gifted by Prof Liling $\mathrm{Wu}$ ) was cultured as previously described (11). ENO1 plasmid (kindly gifted by Prof Feng Chen) and control plasmid transfection was performed with MegeTran 1.0 (Origene, Rockville, MD, USA), and the ratio of transfection reagent to DNA was $3: 1$, as per the manufacturer's instructions. At 24-h post-transfection, cells were collected and treated with azacytidine dissolved in dimethyl sulfoxide. After treatment, cells were lysed with RIPA lysis buffer for $30 \mathrm{~min}$ on ice. The lysates were then centrifuged at $14,000 \times \mathrm{g}$ for $20 \mathrm{~min}$ at $4{ }^{\circ} \mathrm{C}$. The supernatant was collected and the protein concentration was determined using a BCA protein assay kit. Proteomic analysis of the 2 cell groups was the same as previously described.

\section{Statistical analysis}

Statistical analyses were performed using SPSS version 22.0 (SPSS, Chicago, IL, USA). Continuous data were expressed as mean \pm standard deviation, and analyzed using MannWhitney U-test. Results were considered significant when $\mathrm{P}<0.05$.

\section{Results}

\section{Proteomic analysis of proteins in the saliva samples}

Mass spectrometry analysis of saliva samples identified 21 significantly differently expressed proteins $(\geq 2$ - or $\leq 0.5$ fold change) between pSS patients and healthy controls, of which 17 proteins was overexpressed and 4 were underexpressed in pSS patients (Table 1). The expression of ENO1 in pSS saliva was 4.2 times greater than that of the 
Table 1 Salivary proteins differentially expressed in pSS patients compared with healthy controls ( $\geq 2$ - or $\leq 0.5$-fold change)

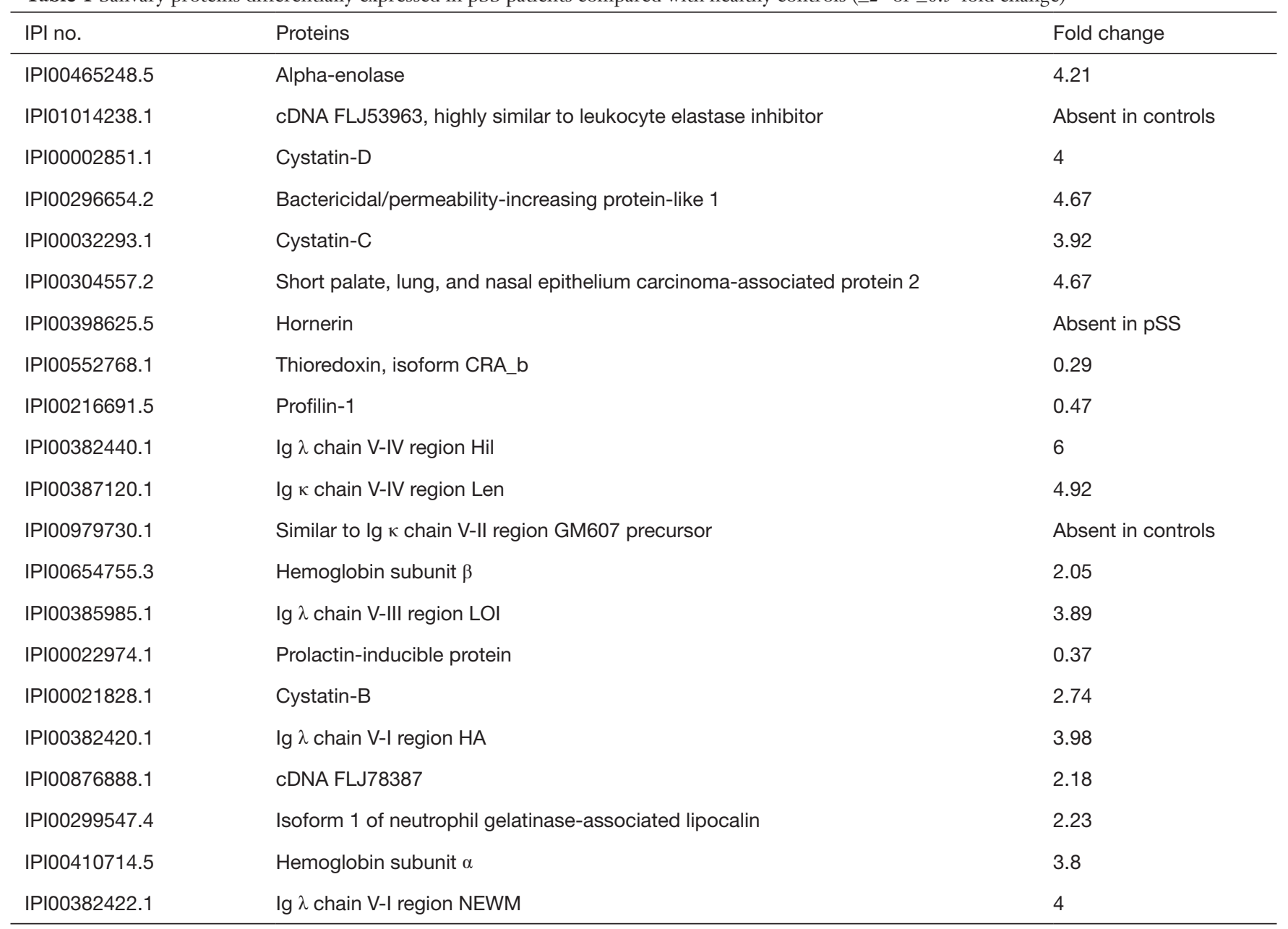

IPI, international protein index. pSS, primary Sjögren's syndrome; Ig, immunoglobulin.

control group.

\section{Verification of ENO1 expression in saliva and labial glands}

ENO1 expression in the saliva samples of the $8 \mathrm{pSS}$ patients and 8 healthy controls was verified by Western blot. As shown in Figure 1, ENO1 was overexpressed in pSS patients compared with healthy controls. We then investigated ENO1 mRNA expression in the labial glands of pSS patients $(n=5)$ and healthy controls $(n=4)$. Reverse transcription-PCR showed that ENO1 mRNA was significantly overexpressed in pSS labial glands compared with those of healthy controls $(\mathrm{P}<0.01)$ (Figure 2). IHC staining of labial glands showed that ENO1 proteins were

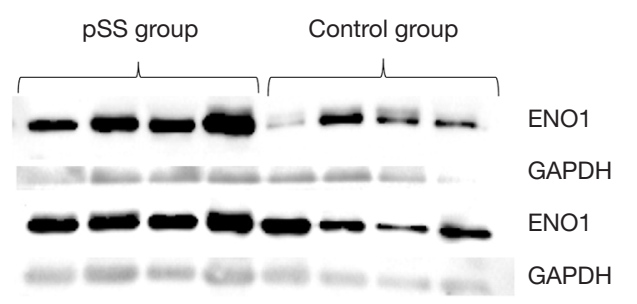

Figure 1 Western blot analysis of salivary ENO1 in saliva from pSS patients $(n=8)$ and healthy controls $(n=8)$. Results indicate that the salivary ENO1 level was much higher in pSS patients compared with healthy controls. ENO1, $\alpha$-enolase-1; pSS, primary Sjögren's syndrome; GAPDH, glyceraldehyde 3-phosphate dehydrogenase. 


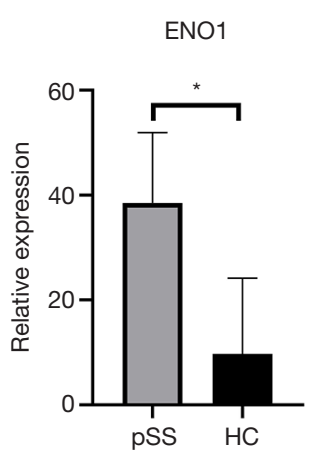

Figure 2 Reverse transcription-polymerase chain reaction analysis of ENO1 mRNA in the labial glands of pSS patients $(n=5)$ and healthy controls $(\mathrm{HC} ; \mathrm{n}=4)$. Results showed that ENO1 mRNA was significantly overexpressed in the labial glands of pSS patients compared with healthy controls. ${ }^{*} \mathrm{P}<0.05$. ENO1, $\alpha$-enolase-1; pSS, primary Sjögren's syndrome; HC, healthy controls. highly expressed in the acinar and duct epithelial cells in pSS patients compared with those of healthy controls. ENO1 was not expressed in infiltrated lymphocytes (Figure 3).

\section{Investigation of ENO1 autoantibody in pSS serum}

We then detected the IgG ENO1 autoantibodies in serum samples of pSS patients $(n=26)$ and healthy controls $(n=20)$ using ELISA. As shown in Figure $4 A$, levels of serum antiENO1 autoantibody were significantly higher in pSS patients $(19.88 \pm 13.77 \mathrm{ng} / \mathrm{mL})$ compared with healthy controls $(8.7 \pm 4.4 \mathrm{ng} / \mathrm{mL})(\mathrm{P}<0.01)$. As shown in Figure $4 B$, the receiver-operating characteristic (ROC) curve analysis was performed to evaluate the performance of anti-ENO1 in distinguishing pSS from healthy controls. The area under the curve (AUC) values of anti-ENO1 was 0.811 .
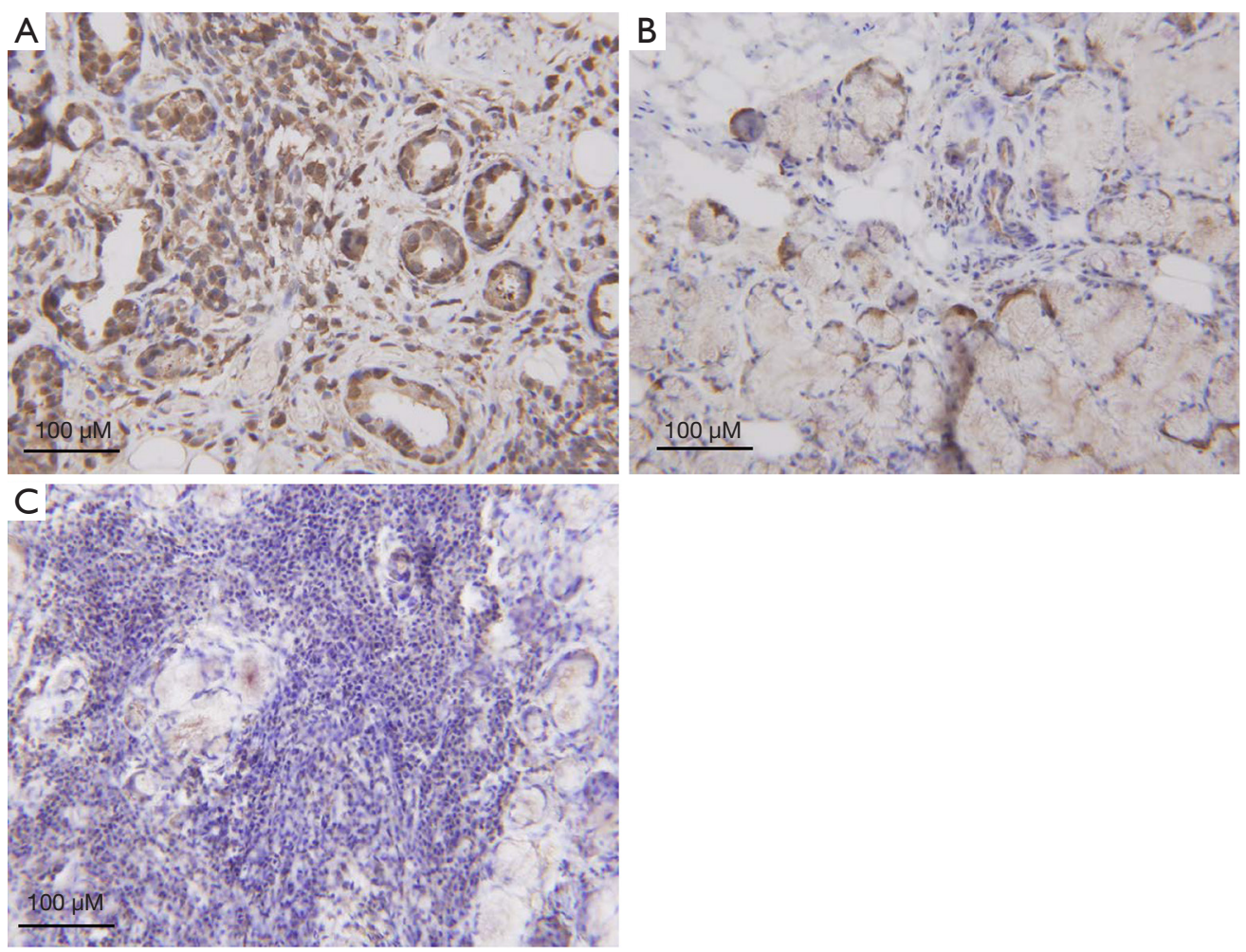

Figure 3 Immunohistochemical staining of labial glands. (A) ENO1 proteins were highly expressed in the acinar and duct epithelial cells in pSS patients. (B) ENO1 expression was lower in healthy controls. (C) ENO1 was not expressed in infiltrated lymphocytes. ENO1, $\alpha$-enolase-1; pSS, primary Sjögren's syndrome. The scale is 1:100 $\mu \mathrm{m}$. 

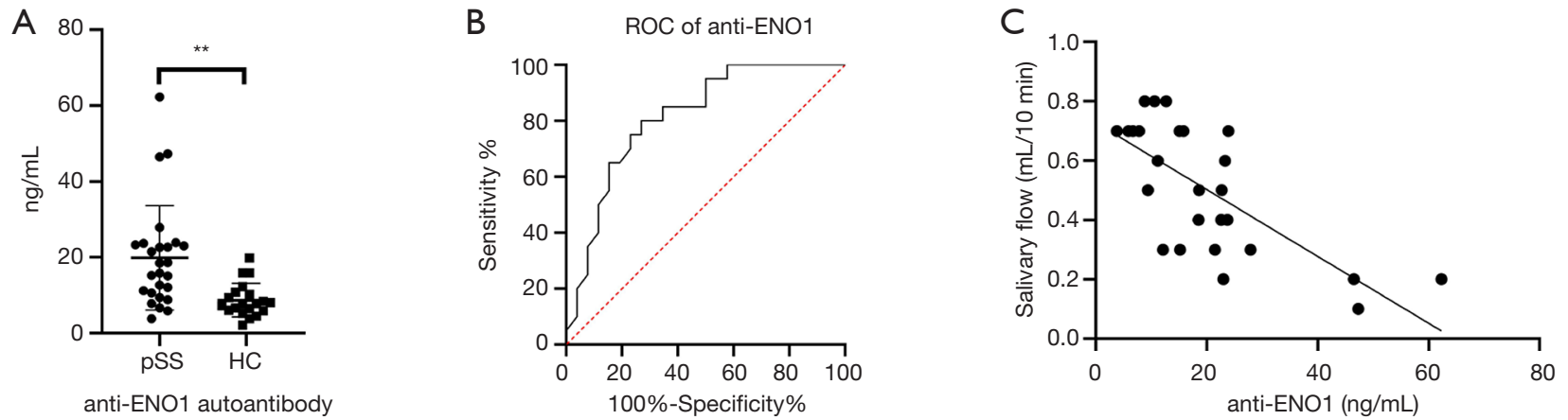

Figure 4 Analyses of anti-ENO1 autoantibody in serum samples from pSS patients and healthy controls. (A) Levels of serum anti-ENO1 autoantibody were significantly higher in pSS patients compared with healthy controls $(\mathrm{P}<0.01)$. (B) ROC curve analysis was performed to evaluate the performance of anti-ENO1 in distinguishing between pSS and healthy controls. Area under the curve value of anti-ENO1 was 0.811. (C) Linear regression analysis showed that anti-ENO1 was moderately associated with salivary flow rate by linear regression (n=26, $\left.\mathrm{R}^{2}=0.5056\right) .{ }^{* *}, \mathrm{P}<0.01$. ENO1, $\alpha$-enolase; pSS, primary Sjögren's syndrome; ROC, receiver-operating characteristic.
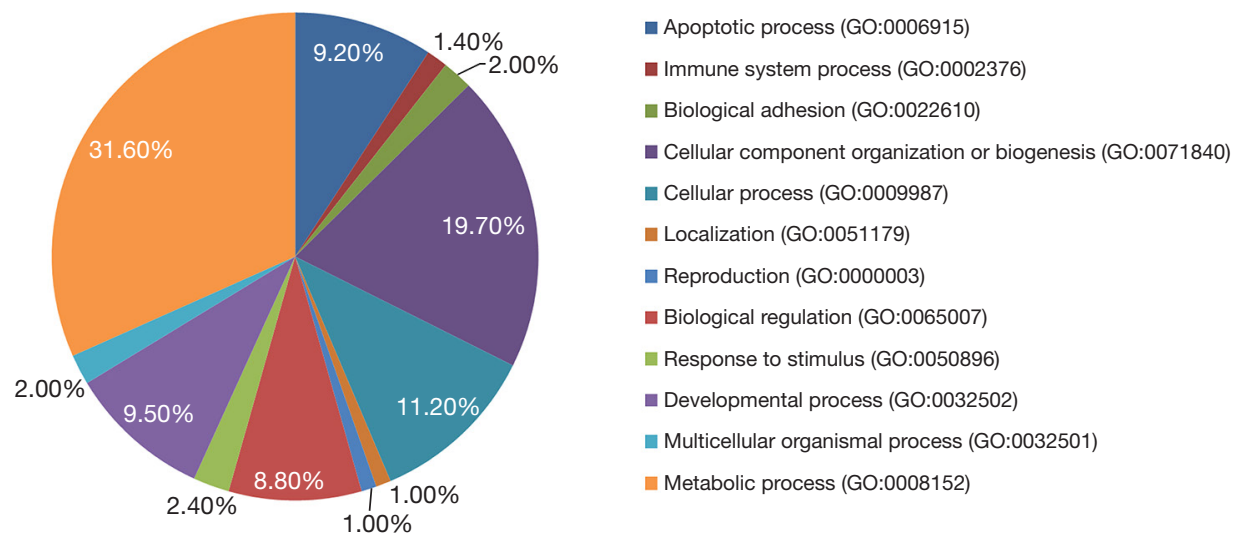

Figure 5 Biologic process of differentially regulated proteins with PANTHER (http://www.pantherdb.org).

Furthermore, linear regression analysis was performed to evaluate the association between anti-ENO1 and salivary flow rates. The results showed that the expression level of anti-ENO1 was moderately associated with salivary flow rate by linear regression $\left(\mathrm{n}=26, \mathrm{R}^{2}=0.5056\right)$ (Figure $\left.4 C\right)$.

\section{Effect of ENO1 overexpression on SMG-C6 cells}

After 24-h transfection, the expressions of total proteins were analyzed by mass spectrometry. The results showed that the expression level of ENO1 in SMG-C6 cells transfected with the ENO1 recombinant plasmid was significantly higher ( $>1.5$-fold) than that of the control group. The results also revealed 199 differentially expressed proteins between the 2 groups, of which 188 proteins were overexpressed in ENO1 transfected SMG-C6 cells $(>1.5$-fold) and 11 proteins were overexpressed in the control group ( $>1.5$-fold). PATHER analysis demonstrated that these differentially expressed proteins participate in diverse biologic processes, including apoptosis, immune system processes, and biologic adhesion (Figure 5).

\section{Discussion}

Primary Sjögren's syndrome (pSS) is identified as a chronic autoimmune disease characterized by exocrine dysfunction and sicca syndrome. Most recently, it has been designated as an autoimmune epithelitis (12). The pathogenesis of pSS is so complex that, despite previously published studies investigating its associated factors, its etiology is still 
poorly understood. Conventionally, risk genes, innate and adaptive immune responses, viral infections, and hormonal factors have been implicated in causation of pSS. In recent years, studies on both immune and non-immune roles of salivary gland epithelial cells (SGEC) have added to the understanding of the disease mechanisms $(13,14)$. Because saliva is secreted directly by salivary glands, it may directly reflect the biologic status of SGEC. In the present study, to identify specific pSS biomarkers, we performed a proteomic analysis on saliva samples from both pSS patients and healthy controls.

In recent years, scientific efforts have been directed toward evaluate salivary proteome to diagnose or monitor pSS (15). Most of these studies emphasize salivary diagnostics. However, the proteolysis, degradation, as well as dephosphorylation of salivary proteins, result in inconsistency of the results among studies. In our study, the mass spectrometry analysis of unstimulated whole saliva revealed 21 differentially expressed proteins between pSS patients and healthy controls, of which the expression level of ENO1 in pSS was 4.21 times greater than that of healthy controls. This result was consistent with previous research (16-18). We then verified this result in saliva samples from additional 8 pSS patients and 8 healthy controls using Western blot. Furthermore, we revealed that ENO1 mRNA was overexpressed in pSS labial glands compared with that of healthy controls, and overexpressed ENO1 was mainly located in the acinar and duct epithelial cells. Hjelmervik et al. also reported that ENO1 is overexpressed in minor salivary glands in pSS patients using the proteomic method (19). However, to the best of our knowledge, our study is the first to explore the ENO1 mRNA expression level and distribution of ENO1 in pSS minor salivary glands.

It has been widely accepted that ENO1 is a highly conserved glycolytic enzyme that plays an important role in cellular energy metabolism (20). However, recent studies have indicated that ENO1 plays a crucial role in a variety of biologic and pathophysiological processes related to both cancer and autoimmune diseases. The aberrant expression of ENO1 has been reported to be associated with multiple tumor progression and is correlated with poor prognosis (21-24). There have been several reports on autoimmune diseases that have indicated that the overexpression of ENO1 could induce and promote inflammation reactions. Bae $e t a l$. reported that ENO1 expressed on the surface of monocytes and macrophages could induce robust synovial inflammation in rheumatoid arthritis (25). Increased cell- surface expression of ENO1 also could promote the migration of inflammatory cells into inflammatory sites (26). Kinloch et al. immunized DR4-IE-transgenic mice with Porphyromonas gingivalis ENO1, and found it could induce autoimmunity to mammalian ENO1 and arthritis (27). Bruschi et al. identified ENO1 as a glomerular target antigen, and found that the interaction with serum ENO1 autoantibodies could induce immunological inflammatory reactions (28). In addition, it has been demonstrated that ENO1 is the most abundant glycolytic enzyme in the apoptotic cell membrane, which could promote autoimmune modulation and induce autoantibodies (29). To investigate the effect of ENO1 overexpression on salivary epithelial cells in pSS, we performed the in vitro SMG-C6 experiment. The results showed that the overexpression of ENO1 could upregulate the expression of several proteins, including signal transducer and activator of transcription 3 (STAT3), which is involved in apoptosis and the immune regulation process, and Zonula occludens-1/2 (ZO-1/2), which is involved in the saliva secretion process. Previously published studies have demonstrated that the disruption of the STAT3 signaling pathway in epithelial cells could induce SS-like autoimmune disease (30). The effect of ENO1 overexpression on immunoreactions and salivary secretion warrants further study.

Previously published studies have discovered ENO1 autoantibody expression in both organ-specific and nonorgan-specific autoimmune diseases (31), including rheumatoid arthritis (6-66\%), systemic lupus erythematosus $(19-80 \%)$, and systemic sclerosis $(15-30 \%)$. ENO1 is widely expressed in prokaryotic and eukaryotic organisms, such as Helicobacter pylori and Porphyromonas gingivalis $(27,32)$. As ENO1 is highly conserved in organisms from bacteria to human, infections of these bacteria could induce a cross-autoimmune response against human ENO1, and lead to the production of ENO1 autoantibody. In addition, the polypeptides of ENO1 broken down by caspase- 1 in autoimmune response, as well as ENO1 expressed in the apoptotic cell membrane, could induce the expression of ENO1 autoantibody. In the present study, we detected the ENO1 autoantibody in serum samples of pSS patients $(19.88 \pm 13.77 \mathrm{ng} / \mathrm{mL})$, and found that it was significantly higher than that of healthy controls $(8.7 \pm 4.4 \mathrm{ng} / \mathrm{mL})$, $\mathrm{P}<0.01$. The ROC curve analysis of ENO1 autoantibody in distinguishing pSS from healthy controls showed that the AUC value was 0.811 . These results were consistent with those of Cui et al. (33). In their study, they identified 3 novel salivary antibodies, including ENO1 autoantibody. They 
found that the expression level of ENO1 autoantibody in pSS saliva was significantly higher than that of healthy controls. The AUC value was 0.82 for distinguishing between pSS and healthy controls. Furthermore, IgG antibody level against citrullinated ENO1 was found to be higher in pSS patients than in rheumatoid arthritis patients and healthy controls $(\mathrm{P}<0.003$ and $\mathrm{P}<0.0001$, respectively). We then analyzed the correlation between ENO1 autoantibody and salivary flow rate, and found only a moderate correlation $\left(\mathrm{R}^{2}=0.5056\right)$. It was also reported that there was no correlation between ENO1 autoantibody and clinical manifestations and other laboratory parameters in previous studies $(34,35)$. However, ENO1 autoantibody level has been reported to be related to bone erosion and rheumatoid arthritis-associated interstitial lung disease (36), and with Hashimoto's encephalopathy and glomerulonephritis in autoimmune thyroiditis $(37,38)$. Compared with target organ-specific antigens, ENO1 is expressed more widely in certainly tissues, such as vascular endothelium and neurons. The autoimmune response to ENO1 is more strongly associated with lesions outside the target organ. Future studies with more pSS patients with extraglandular involvement are warranted to evaluate the correlation between ENO1 autoantibody and clinical manifestations.

The findings of the present study indicated that ENO1 was overexpressed both in the saliva and labial glands of pSS patients, and induced the production of IgG ENO1 autoantibody. The expression level of serum ENO1Ab could distinguish between pSS and healthy controls, and was moderately correlated with the salivary flow rate. The overexpression of ENO1 could upregulate a number of proteins involved in apoptosis and the immune regulation process. However, the precise role of ENO1 in the pathogenesis of pSS warrants further study.

\section{Acknowledgments}

Funding: This work was supported by Beijing Natural Science Foundation (no. 7204331).

\section{Footnote}

Reporting Checklist: The authors have completed the MDAR checklist. Available at http://dx.doi.org/10.21037/gs-20-814

Data Sharing Statement: Available at http://dx.doi. org/10.21037/gs-20-814
Conflicts of Interest: All authors have completed the ICMJE uniform disclosure form (available at http://dx.doi. org/10.21037/gs-20-814). The authors have no conflicts of interest to declare.

Ethical Statement: The authors are accountable for all aspects of the work in ensuring that questions related to the accuracy or integrity of any part of the work are appropriately investigated and resolved. All procedures performed in this study involving human participants were in accordance with the Declaration of Helsinki (as revised in 2013). The present study was approved by the Biomedical Ethics Committee of Peking University School of Stomatology (Beijing, China). All participants provided signed informed consent.

Open Access Statement: This is an Open Access article distributed in accordance with the Creative Commons Attribution-NonCommercial-NoDerivs 4.0 International License (CC BY-NC-ND 4.0), which permits the noncommercial replication and distribution of the article with the strict proviso that no changes or edits are made and the original work is properly cited (including links to both the formal publication through the relevant DOI and the license). See: https://creativecommons.org/licenses/by-nc-nd/4.0/.

\section{References}

1. Al-Hashimi I, Khuder S, Haghighat N, et al. Frequency and predictive value of the clinical manifestations in Sjogren's syndrome. J Oral Pathol Med 2001;30:1-6.

2. Loustaud-Ratti V, Riche A, Liozon E, et al. Prevalence and characteristics of Sjgren's syndrome or Sicca syndrome in chronic hepatitis $\mathrm{C}$ virus infection: a prospective study. J Rheumatol 2001;28:2245-51.

3. Fox RI. Sjögren's syndrome. Lancet 2005;366:321-31.

4. Kassan SS, Moutsopoulos HM. Clinical manifestations and early diagnosis of Sjögren syndrome. Arch Intern Med 2004; 164:1275-84.

5. Elkon K, Casali P. Nature and functions of autoantibodies. Nat Clin Pract Rheumatol 2008;4:491-8.

6. Vitali C, Bombardieri S, Jonsson R, et al. Classification criteria for Sjogren's syndrome: a revised version of the European criteria proposed by the American-European Consensus Group. Ann Rheum Dis 2002;61:554-8.

7. Fayyaz A, Kurien BT, Scofield RH. Autoantibodies in Sjogren's Syndrome. Rheum Dis Clin North Am 2016;42:419-34. 
8. Lee JM, Garon E, Wong DT. Salivary diagnostics. Orthod Craniofac Res 2009;12:206-11.

9. Wei P, Li C, Qiang L, et al. Role of salivary anti-SSA/ $B$ antibodies for diagnosing primary Sjögren's syndrome. Med Oral Patol Oral Cir Bucal 2015;20:e156-60.

10. Tang H, Tian E, Liu C, et al. Oxidative stress induces monocyte necrosis with enrichment of cell-bound albumin and overexpression of endoplasmic reticulum and mitochondrial chaperones. PLoS One 2013;8:e59610.

11. Xiang RL, Mei M, Cong X, et al. Claudin-4 is required for AMPK-modulated paracellular permeability in submandibular gland cells. J Mol Cell Biol 2014;6:486-97.

12. Mitsias DI, Kapsogeorgou EK, Moutsopoulos HM. Sjögren's syndrome: why autoimmune epithelitis? Oral Dis 2006;12:523-32.

13. Sandhya P, Theyilamannil Kurien B, Danda D, et al. Update on pathogenesis of Sjogren's syndrome. Curr Rheumatol Rev 2017;13:5-22.

14. Manoussakis MN, Kapsogeorgou EK. The role of intrinsic epithelial activation in the pathogenesis of Sjögren's syndrome. J Autoimmun 2010;35:219-24.

15. Katsiougiannis S, Wong DT. The proteomics of saliva in Sjögren's syndrome. Rheum Dis Clin North Am 2016;42:449-56.

16. Baldini C, Giusti L, Ciregia F, et al. Proteomic analysis of saliva: a unique tool to distinguish primary Sjögren's syndrome from secondary Sjögren's syndrome and other sicca syndromes. Arthritis Res Ther 2011;13:R194.

17. Hu S, Gao K, Pollard R, et al. Preclinical validation of salivary biomarkers for primary Sjögren's syndrome. Arthritis Care Res (Hoboken) 2010;62:1633-8.

18. Hu S, Wang J, Meijer J, et al. Salivary proteomic and genomic biomarkers for primary Sjögren's syndrome. Arthritis Rheum 2007;56:3588-600.

19. Hjelmervik TO, Jonsson R, Bolstad AI. The minor salivary gland proteome in Sjögren's syndrome. Oral Diseases 2009;15:342-53.

20. Ejeskär K, Krona C, Carén H, et al. Introduction of in vitro transcribed ENO1 mRNA into neuroblastoma cells induces cell death. BMC Cancer 2005;5:161.

21. Cancemi P, Buttacavoli M, Roz E, et al. Expression of alpha-enolase (ENO1), Myc promoter-binding protein-1 (MBP-1) and matrix metalloproteinases (MMP-2 and MMP-9) reflect the nature and aggressiveness of breast tumors. Int J Mol Sci 2019;20:3952.

22. Zhan P, Zhao S, Yan H, et al. $\alpha$-enolase promotes tumorigenesis and metastasis via regulating AMPK/ mTOR pathway in colorectal cancer. Mol Carcinog
2017;56:1427-37.

23. Sun L, Guo C, Cao J, et al. Over-Expression of AlphaEnolase as a Prognostic Biomarker in Patients with Pancreatic Cancer. Int J Med Sci 2017;14:655-61.

24. Sun L, Lu T, Tian K, et al. Alpha-enolase promotes gastric cancer cell proliferation and metastasis via regulating AKT signaling pathway. Eur J Pharmacol 2019;845:8-15.

25. Bae S, Kim H, Lee N, et al. $\alpha$-Enolase expressed on the surfaces of monocytes and macrophages induces robust synovial inflammation in rheumatoid arthritis. J Immunol 2012;189:365-72.

26. Wygrecka M, Marsh LM, Morty RE, et al. Enolase-1 promotes plasminogen-mediated recruitment of monocytes to the acutely inflamed lung. Blood 2009;113:5588-98.

27. Kinloch AJ, Alzabin S, Brintnell W, et al. Immunization with Porphyromonas gingivalis enolase induces autoimmunity to mammalian $\alpha$-enolase and arthritis in DR4-IE-transgenic mice. Arthritis Rheum 2011;63:3818-23.

28. Bruschi M, Sinico RA, Moroni G, et al. Glomerular autoimmune multicomponents of human lupus nephritis in vivo: $\alpha$-enolase and annexin AI. J Am Soc Nephrol 2014;25:2483-98.

29. Ucker DS, Jain MR, Pattabiraman G, et al. Externalized glycolytic enzymes are novel, conserved, and early biomarkers of apoptosis. J Biol Chem 2012;287:10325-43.

30. Okuma A, Hoshino K, Ohba T, et al. Enhanced apoptosis by disruption of the STAT3-IкB- $\zeta$ signaling pathway in epithelial cells induces Sjögren's syndrome-like autoimmune disease. Immunity 2013;38:450-60.

31. Terrier B, Degand N, Guilpain P, et al. Alpha-enolase: a target of antibodies in infectious and autoimmune diseases. Autoimmun Rev 2007;6:176-82.

32. López-López MJ, Rodríguez-Luna IC, Lara-Ramírez EE, et al. Biochemical and Biophysical Characterization of the Enolase from Helicobacter pylori. Biomed Res Int 2018;2018:9538193.

33. Cui L, Elzakra N, Xu S, et al. Investigation of three potential autoantibodies in Sjogren's syndrome and associated MALT lymphoma. Oncotarget 2017;8:30039.

34. Olivares-Martínez E, Hernández-Ramírez DF, NúñezÁlvarez CA, et al. $\alpha$-enolase is an antigenic target in primary Sjögren's syndrome. Clin Exp Rheumatol 2019;37:29-35.

35. Nezos A, Cinoku I, Mavragani CP, et al. Antibodies against citrullinated alpha enolase peptides in primary Sjogren's syndrome. Clin Immunol 2017;183:300-3.

36. Alunno A, Bistoni O, Pratesi F, et al. Anti-citrullinated 
alpha enolase antibodies, interstitial lung disease and bone erosion in rheumatoid arthritis. Rheumatology (Oxford) 2018;57:850-5.

37. Liu H, Shan Z, Li C, et al. Maternal subclinical hypothyroidism, thyroid autoimmunity, and the risk of miscarriage: a prospective cohort study. Thyroid
2014;24:1642-9.

38. Koçak G, Huddam B, Azak A, et al. Coexistent findings of renal glomerular disease with Hashimoto's thyroiditis. Clin Endocrinol (Oxf) 2012;76:759-62.

(English Language Editor: R. Scott)
Cite this article as: Wei P, Xing Y, Li B, Chen F, Hua H. Proteomics-based analysis indicating $\alpha$-enolase as a potential biomarker in primary Sjögren's syndrome. Gland Surg 2020;9(6):2054-2063. doi: 10.21037/gs-20-814 